\author{
Proceedings of the \\ $1^{\text {st }}$ International Conference and Exhibition on Future RFID Technologies \\ Eszterhazy Karoly University of Applied Sciences and \\ Bay Zoltán Nonprofit Ltd. for Applied Research \\ Eger, Hungary, November 5-7, 2014. pp. 101-113 \\ doi: 10.17048/FutureRFID.1.2014.101
}

\title{
Definition of digital ownership, digital data treasure, informationbank, some use cases and a business model*
}

\author{
Gábor Kusper \\ Eszterhazy Karoly University of Applied Sciences, Eger, Hungary \\ gkusper@aries .ektf .hu
}

\begin{abstract}
By digital ownership we mean that we have permission to access and/or use data, information and knowledge about ourselves or about the objects we own. We can also grant or revoke rights on these data. Digital ownership is a layer of Internet of Things (IoT). IoT enables to gather gigabytes of data on a single thing. Digital ownership helps us to gain control on these huge data. One of the pre-requests of digital ownership is that the object must be identifiable uniquely, for example by an EPC code, and the stored data must be accessible. The sum of the stored data about our objects is called digital data treasure. Informationbank is the storage of digital data treasure. An informationbank could be a passive or active actor of the IoT infrastructure. Active informationbanks compete with each other to collect digital data treasure of humans and companies. They make money out of derived data mined from the digital data treasure, but they are not allowed to sell the data treasure, only derived and anonymous data. They also share their income with data sources, like the producer of a product, who submit information about the creation of its products. We also present some use cases which underline how useful the digital owner-ship.
\end{abstract}

${ }^{*}$ This research was realized with the support of the European Union, co-financed by the European Social Fund within the framework of the project "Internet of things" along the concept TÁMOP-4.2.2.C-11/1/KONV-2012-0014 FutureRFID - Further development of RFID/NFC technology. 


\section{Introduction}

In this article we analyze the concept of the Internet of Things (IoT) in the light of digital ownership. At first we review the best known definitions of the IoT, as well as the techniques needed to implement it. Then we give an own definition by which the IoT is an infrastructure which enables digital ownership. In this article we introduce several new concepts:

- Digital ownership: Under digital ownership we understand the ability to access the information and knowledge of physically owned objects at anytime, anywhere, and to use them and control the access of other users to it.

- Digital data treasure: All saved information of our objects is called digital data treasure, or shortly data treasure.

- Information bank or shortly infobank: We call the storage of digital treasure infobank.

We base the statements of the new concepts in their chapters, theories, which, in our view, help to make the practice of digital ownership possible, enabled by the IoT infrastructure.

Giving the IoT concept an own definition doesn't mean rejecting the already existing concept of the IoT. Rather, it means giving the concept of the IoT a new layer.

\section{A quick overview of the IoT}

Everyone agrees that the concept of the IoT was used by Kevin Ashton in 1999 the first time (based on other sources in 1998). In one of his later works [1] the following reasoning can be read out (not literally): the approximately 50 PetaByte on the internet where created by humans, humans took photos, typed text, pressed the button. At the same time humans are not able to upload more data than that because they get exhausted and find uploading boring. Because of this they will not agree to record more and more data from the physical world to upload it to the internet. Because of this we have to trust machines, objects to do this work since having every measurable data on the internet quickly and correctly has huge advantages for us, e.g. we could decrease excess production. We would know exactly when to replace or repair an object. We would know which product is fresh, and which is depraved. The Internet of Things can change the world, just as the internet has changed it.

It is apparent that Kevin Ashton sees a very useful tool in the IoT. Insomuch that he has helped to create the Auto-ID Center research lab on the MIT university in 1999. From this laboratory the international re-search network called Auto-ID Labs (www.autoidlabs.org) was formed, of which several renowned uni-versities and research labs became members. Finally a network called EPCglobal was established (www.epcglobal.org) in 2003, which had the purpose of establishing the 
Electronic Product Code ${ }^{\mathrm{TM}}$, EPC and the standards associated with it. Formally the International Telecommunication Union (ITU) named it in a document called "ITU Internet report" in 2005. The next important document is an article called "Vision and challenges for realizing the Internet of Things" published by CERPIoT [13] in 2010, which lists the technologies required for the implementation of the IoT. We list some of the outstanding definitions of the IoT:

- The definition found in article [1]: "The Internet of Things has the potential to change the world, just as the Internet did. Maybe even more so."

- The definition found in article [14]: "Things having identities and virtual personalities operating in smart spaces using intelligent interfaces to connect and communicate within social, environmental, and user contexts."

- The definition found in article [15]: "The semantic origin of the expression is composed by two words and concepts: "Internet" and "Thing", where "Internet" communication protocol, the Internet suite (TCP/IP)", while "Thing" is "an object not precisely identifiable" Therefore, semantically, "Internet of Things" means "a world-wide network of interconnected objects uniquely addressable, based on standard communication protocols."

- The definition found in article [16]: "The Internet of Things allows people and things to be connected anytime, anyplace, with anything and anyone, ideally using any path/network and any service."

In the area of the IoT some review articles exist which describe in detail, who defined this concept until then and how. Of these we reviewed two articles $[2,11]$ in detail. According to the first review article [2] the concept of the IoT has 3 approaches: object-oriented, network-oriented and meaning-oriented.

The EPC [3, 4] mentioned so far is the object oriented approach to the IoT, between those it is perhaps elaborated the most. The main rival is the uID system [5], where the "u" letter refers to these three English words: unique, universal, ubiquitous. The uID system's purpose is the global traceability of objects.

Under the network-oriented approaches the Web of Things [6] is probably the best known according to which computers have to be embedded in objects and connected them to the internet with existing technologies.

Among the network-oriented approaches probably the best known is the Web of Things [6] where com-puters have to be embedded into the objects and connected to the internet with the help of existing technologies. For this it is necessary that we switch from IPv4 to IPv6 as we are slowly running out of the possible 4.3 milliard IP addresses. Another network-oriented approach is IPSO (IP for Smart Objects) Alliance [7], an alliance which was established with the purpose to work out a recommendation which helps the TCP/IP protocol to be able to connect batterypowered smart objects. The internet $\varnothing[8]$ had the same purpose as well, which sees the reach of "IP over anything" idea in the simplification of the IP protocol.

As the approaches mentioned until now deal with the Internet of Things with the focus on things or the internet, the meaning- or semantics-oriented point of 
view sees its main task in the conversion of great amounts of data to information. This is consistent with the Big Data problem, with the difference that we know which data belongs to which object.

In the article [9] we can read a proposal where we have to use the technology semantic web for the semantic approach to the IoT, like the RDF, OWL and WSML languages which are able to describe an-thology for example. This article also devotes space to what kind of development is needed for the EPC to comply with the semantics-oriented point of view. For instance we can see a recommendation, which states that we need semantic extensions for the Product Markup Language (PML) which belongs to the standards developed by EPCGlobal.

In the article [10] we see a recommendation which states that we need to rely on semantic technologies for the filtering, processing of data. An example for this is the agent technology, the automatic recognition of topics, terms, the extraction of information and meaning, as well as the categorization. There is a need for this as humans will not be able to deal with this great amount of data, so they have to utilize intelligent assistants.

The newest article which has a summary of the IoT is probably article [11] which sees the IoT as the next natural step in the development of the internet. At the beginning the internet only connected several computes, it was possible to send emails, and download and upload files with the help of ftp. The next step included the WWW with more and more websites and services. After that the era of the mobile internet began, where it was possible to reach the internet from mobile phones and other portable devices. The next step was where people were connecting to the internet with the help of social networks. The next step in the development of the internet is that in addition to servers, clients, mobile devices and humans, objects are able to connect to the internet. That's what we call the IoT.

Another finding of article [11] is that a lot of intermediate layers are needed because of the great amount of data which, context aware, are able to preprocess data.

\section{Digital ownership}

In the previous chapter we reviewed the different definitions of the IoT. Our own definition is the following: the IoT is an infrastructure which makes the practice of digital ownership possible.

This definition, as well as all the other definitions we could see in the previous chapter, helps us to un-derstand the concept of the IoT, although this definition does not describe the term of the IoT as all the other definitions don't do either. Therefore we need to regard this definition as an extension to all the other definitions.

The fundamental question of digital ownership is that if I buy an object (or service), does the associated data, information, knowledge belong to me, i.e. do I own the object digitally as well. 
We often hear that we live in an information society where it is natural to communicate quickly and cheaply, where route planning isn't a problem, where the only source of problems can be the searching between such large amounts of data. In this environment the need to access as much information as possible from the objects surrounding us easily and quickly seems natural.

Statement 3.1. In the information society digital ownership is a natural basic right.

The reason for us not being able to easily access information about our own objects, as well as the objects surrounding us is that the appropriate infrastructure has not been formed yet, the IoT infrastructure. This will be the tool with the help of which we will be able to easily access our objects as well as the summary of the data / information / knowledge of the objects we are interested in.

Statement 3.2. The IoT is a tool, with which we can access data of objects which are of interest for us.

It is important to notice that digital ownership doesn't necessarily mean that we own data / information / knowledge associated with our objects in a digital form, in the form of electronic data, although the word digital is primarily used in an electronic sense. Digital ownership can be satisfied with the handover of printed documents.

The easiest way to describe data belonging to an object is by using an example. Let's look at a car for example. When we get it, we get the technical specifications, a service book, and a registration certificate. We own the car digitally because we got the associated data.

In many cases we don't get any data associated with bought items. For instance, when we look at a croissant, we can only inform ourselves from the label on the shelf, but this shows only the price.

Digital ownership is of elementary importance because in our complicated world we often meet items of which we don't have enough information to make necessary decisions regarding the items. For instance, if someone suffers from lactose intolerance it is very important for him/her to know whether milk was used to produce the croissant or not.

Furthermore this information is important even before we own the croissant. This means that in many cases the person or organization having digital ownership have to make it possible that some data gets accessible before the ownership is transferred. Regarding the example above the ingredients have to be made accessible for buyers.

In other cases we need long forgotten knowledge regarding one of our objects. Imagine, soft drink gets poured out on the couch leaving an ugly spot. It is easy to imagine that nobody remembers how to clean a couch. Then digital ownership helps because through it we own the appropriate knowledge and only have to look for it.

Based on the examples above we give the following definition: 
Definition 3.3 (Digital ownership). Under digital ownership we understand that we have the right to access, use and control other's access to all data, information and knowledge about objects we physically own.

\section{Digital data treasure}

In this chapter we deal with the subject of digital ownership, the digital data treasure. At first we give this concept a definition.

Definition 4.1 (Digital data treasure, in short data treasure). The sum of all data owned by digital ownership.

Now that we already know about the data, information and knowledge belonging to objects, the next question would be how we can take possession of them. At the moment the internet, search engines on the internet help us in orientation through searching for the type identifier of objects.

The problem with the type identifier is, that not every product / service / object has one and that a type identifier like for example the name of a product (e.g. Samsung Galaxy S4) does not individually identify an object. Some objects have individual identifiers, like the cars' license plate number or the VIN. Searching for those on the internet usually doesn't have any results.

At the same time during the production of a product a lot of data is produced about a product which is saved by the producers usually. Data which is generated during the production could be important for the user as well. As saw, in the case of food (e.g. a croissant) the ingredients are important as well as of what quality they are.

If every product would be individually identifiable, the buyer could choose much more consciously. At the same time that's not just in the interest of the buyer. For instance, if it turns out that some products are faulty, possibly dangerous, not every product would have to be called back, only those which are affected.

Statement 4.2. If the producer makes the data which was generated during the production accessible, using individual identifiers makes sense.

However, individual identification of food doesn't make sense, because two croissants are completely the same if they are made of the same ingredients (batch). So for food a batch-level identification is enough.

\section{Obtaining digital ownership through purchase}

We previously stated that not every product has a type identifier. A simple solution to this problem would be a QR code / NFC / RFID tag somewhere (in summary: identifier tags), which identifies the product somehow. If we read a QR code, it is usually a URL, so it leads to a website. The same could be ac-complished using the other two technologies. 
They only question which remains is where the identifier tags should be placed. A lot of goods have identifiers on them, e.g. every technical good. On a lot of goods identifiers can't be placed or printed, for instance because we want to eat them. These products usually have a package. The package is com-pletely suitable for the placement of the identifiers but it can get lost and some goods don't even have packages.

The solution is the shelf. Every good has a shelf or a storage / place similar to a shelf (even in the case of a web store) which is able to report data. On the shelf we can see the name of a product, often the producer and the price. Besides that we can feel free to put display an identifier tag.

Statement 5.1. In the case of every product the shelf of the product is suitable for the placement of an iden-tifier tag.

Let's still stay at the purchase of a croissant. Let's take the lucky case where a QR code is at the crois-sant's counter which leads to a website where we can see of which ingredients the croissant was made. When we would need to know which ingredients the croissant was made of, it can simply occur that we don't find the website. We look at the croissant in vain, there is no tag on it, not even on the package as it didn't have a package.

This implies that we have to give the buyer a way to save the croissant's data among his personal data to a place which is easily accessible. This place can be a smart card which is handed over at purchase. It can be a web host which I can easily access and I only have to hand over a URL at purchase where the data should be sent.

Neither one of the two opportunities mentioned is lifelike at the moment. In any case it can be seen that the purchase is the act where the owner of the digital ownership has to be replaced.

\section{Infobank}

In this chapter we deal with the process of the storage, acquisition, utilization and reaping of a digital treasure.

Let's think over where the digital treasure should be saved?

This place could be a smart card, a smart phone or a USB stick. If we choose this solution everyone has to trust himself.

The other solution is the storage of the data on a server or better a cloud. In today's internet we can get even several gigabytes of storage easily. In this case we have to trust a big IT company that they don't misuse our data.

The third solution would be a storage accessible through a client area. For this we have to trust the state.

So the question sounds like this: who do I trust my digital treasure? Myself, a big IT company or the state? 
Our recommendation is a fourth opportunity. Let's trust a so-called information bank, or in short infobank with our digital treasure which we define in the following way.

Definition 6.1 (Information bank, in short: infobank). We call informational systems which are able to store, edit, query a digital treasure information bank, or in short infobank. We call the specialized profit-oriented or nonprofit organizations for the operation of such systems infobank-organizations, in short infobank.

If it would not be clear whether we understand an informational system or an organization under infobank, the full name (infobank system / infobank organization) should be used.

An infobank-system can be privately owned, owned by an organization or in the hand of a state. An infobank system can be quite small which fits on a mobile phone or quite big where a cloud-based computer technology is needed.

Infobanks represent an important part of the IoT as by our definition the IoT supports the practice of digital ownership which assumes the access / usage of the digital data treasure which we store in infobanks.

Statement 6.2. Infobanks are the special elements of the IoT infrastructure.

\section{Should the infobank be active or passive?}

The infobank can be passive or active. We call the infobank passive if it doesn't use the stored data treasure for profit. We call it active if it does. According to another definition the passive infobank doesn't actively collect the data treasure as the active one does.

In both cases the infobank offers useful services like the preservation of the data treasure, guaranteeing safety and a comfortable surface. From the perspective of the IoT infrastructure it doesn't matter if an infobank is passive or active as long as it performs the necessary actions.

So the difference is in using the data for profit. The active infobanks use the data treasure for profit so they sell the anonym information which can be obtained from the data treasure. They can't sell the data treasure itself but information like what percentage of men between 18 and 25 years possesses a car is already tradable. Active banks live from this income.

Passive banks can't sell any data / information / knowledge. They just save them and make them ac-cessible for those who are authorized. These banks maintain themselves from the payments made by users or transaction fees.

Let's look at a case where in a Ford factory a new Ford Fiesta is built. The passive infobank tries to convince the Ford factory to save the data of the Fiesta at it as it lives from the transaction fees. The active infobank tries to convince the factory as well as it is beneficial for it if as much data as possible is saved there since very profitable data can be earned with data mining tools from a lot of data (Big Data). The active bank doesn't ask for a transaction fee because of this. 
Let's think of this from Ford's perspective. Should I upload the new Fiesta's data to any infobank? If there is a legal regulation for it, so it is mandatory to upload it I will upload it but if not, I'm not going to pay for the upload, then I'll better choose the infobank that's free for me and only do that if I have a competitive advantage from that. That is the buyer buys my product more likely if I use the infobank.

Of course a solution for the Ford factory could be to operate a server where data about the Fiesta is stored but at the same time the Ford factory is a car factory, not an IT company.

By this train of thought some statements can be drawn:

- It is in the interest of both, the active and the passive infobank to store as much data as possible.

- Really valuable information can be drawn from a large data treasure, so the active infobanks are more interested in gathering the data treasure.

- The infobanks can be passive if legal regulations requires the manufacturers / distributors / other data sources to save data in infobanks.

- Active infobanks are able to provide very cheap or free services.

In our view passive infobanks will be viable if the law or another regulator requires the use of such. For the spreading of active infobanks a lot of data has to be available for them of which they are able to mine valuable information (interest).

If the infobanks will spread we will likely meet with passive banks at first, and soon, as competition intensifies and the infobanks have to reduce their transaction fees some active infobanks will appear which will truly compete for our data treasure.

\section{Operation of the infobanks}

Infobanks should be imagined as plain banks where we can store our money and other values but in these banks we store our data treasure. Infobanks can be active or passive players. For both types it is true that if it comes to our mind to save data, we can save this data in the infobank. If we need the data we can take our data from the infobank. Here is the first difference compared to a real bank: if I take data out, it doesn't have to necessarily be removed from the bank. Indeed, this is the recommended usage.

The second difference is that the data is bound to an object (of course there can be data which is not bound to an object in the infobank, but this doesn't matter in our present train of thoughts), so if the ownership of the object changes the ownership of the data changes as well. This means that if I sell my car I also sell the digital data treasure which is belonging to the car.

The third type of usage is completely identical with the usage of an ordinary bank. When I would like to access data the infobank checks whether I have the 
right to do that or not. As accessing doesn't mean deterioration of the data, the question whether others and not only the owner are allowed to access the data treasure, rightfully arises.

Statement 8.1. The authorization has to be checked at every transaction by the infobank.

If we look at the process, the first step is the placement of the data. The infobank has to be notified which data belongs to which object. For this the object has to possess an individual, batch or type identifier. Note that it's not the task of the infobanks to create identifiers. This is ensured by the other elements of the IoT infrastructure like e.g. in the case of the EPC standard the EPC manager [4].

Comment. The EPC manager is an organization which has been given authorization by an issuing agency to issue EPC identifiers to an EPC identifier interval. It is its duty to provide the uniqueness of the EPC identifiers issued by it. Often the EPC manager performs the commissioning which means the binding of the EPC identifier to an object. For more information see [4].

Beyond the object identifier I have to provide the information in which role I would like to upload information about the object. Beyond this I have to prove that I am authorized for this role. If for instance I'd like to upload information as an owner, I have to prove my ownership.

The data treasure can be used with a data treasure identifier. If we only know the identifier of the object we don't accomplish anything with that. Respectively we accomplish that we can create another data treasure belonging to the object if I prove that I am authorized for an appropriate role (e.g. a producer for an object). Then the two data treasures are not completely independent from each other. The owner of the object accesses any data of the other data treasures as well which belongs to the object. At the same time he can't change it, only use it. The other roles (not the owners) usually can't access data from the other data treasures.

Statement 8.2. Multiple data treasure-records can belong to an object, usually one for each role.

\section{Solutions similar to infobanks}

In this chapter we have a look at knowledge base systems similar to infobanks. These systems store information not only belonging to objects but also to humans, creatures, concepts. The root of knowledge base systems is Marvin Minsky's common sense concept $[17,18]$. The purpose of knowledge base projects is the record, storage of knowledge belonging to common sense and making it searchable; sim-ilarly to the infobanks.

The Open Mind Common Sense project: Multiple common sense projects are known. Among those the Open Mind Common Sense project [19] which functions under the supervision of the MIT, is probably the most-known. This project 
started in 1999 and since then more than one million English phrases belonging to common sense were gathered. These are used by the ConceptNet semantic network.

OpenCyc 2.0 system: The OpenCyc 2.0 system [20] is accessible since July 2009. 47000 terms and 306000 statements can be found in the database. OpenCyc is the freely accessible version of the non-free Cyc technology. The ontology behind it is downloadable in the OWL format.

Wolfram Alpha system: The Wolfram Alpha system is a question-answering system which was devel-oped by the company Wolfram Research which also developed the well-known Methematica system. The system is based on relatively few rules but on more than a thousand databases. (e.g. food-ingredients, wisecracks, maps, ....).

YAGO2: YAGO2 [21] is a semantic knowledge base, which gets its stored knowledge from Wikipedia, WordNet and GeoName services. That is, while behind Wolfram Alpha there are a lot of specific databases, behind this there are only three rather general ones, although GeoNames clearly contains geographic data. The collected data includes 10 million terms and 460 million facts.

DBpedia: The DBpedia [22] system downloads structured information from the Wikipedia service as well. DPpedia's biggest contribution to the field is the development of the SPARQL query language with the help of which it is possible to gain information from the database.

\section{Summary}

This article reveals the conceptual system of digital ownership to us. It includes a lot of statements which can provoke controversy. We believe that these discussions will help with the construction of a working IoT infrastructure which makes the achievement of digital ownership possible.

We didn't cover some important and interesting questions in this present article. For instance, how the selling of ownership should be handled, since an object is sellable, so digital ownership has to be handed over as well.

A lot of economic considerations had a place in the article as well, for instance, whether infobanks should be passive or active.

In the future we would like to create white paper which describes the informational system which manages the infobanks and the digital data treasures.

\section{References}

[1] K. Ashton (2009): That 'Internet of Things' Thing, in the real world things matter more than ideas. RFID Journal. June 22, 2009. 
[2] L. Atzori, A. Iera, G. Morabito (2010): The Internet of Things: A survey, Computer Networks 54, pp. 2787-2805. 2010.

[3] K.S. Leong, M.L. Ng, D.W. Engels (2005): EPC Network Architecture, Auto-ID Labs, White Paper Series, http://autoidlabs.org/publications, pp. 1-14. 2005.

[4] Ken Traub (editor) (2013): The GS1 EPCglobal Architecture Framework, GS1 Final Version 1.5, http://www.gs1.org/gsmp/kc/epcglobal/architecture, Approved 23 March 2013.

[5] K. Sakamura (2006): Challenges in the age of ubiquitous computing: a case study of T-engine - an open development platform for embedded systems. Proceedings of ICSE'06, Shanghai, May 2006.

[6] D. Guinard, T. Vlad (2009): Towards the web of things: web mashups for embedded devices. Pro-ceedings of the International World Wide Web Conference 2009, Madrid, Spain, April 2009.

[7] A. Dunkels, J.P. Vasseur (2008): IP for Smart Objects, Internet Protocol for Smart Objects (IPSO) Alliance, White Paper \#1, http: //www .ipso-alliance.org/, September 2008.

[8] N. Gershenfeld, R. Krikorian, D. Cohen (2004): The internet of things, Scientific American 291:4, pp. 76-81. 2004.

[9] I. Toma, E. Simperl, G. Hench (2009): A joint roadmap for semantic technologies and the internet of things. Proceedings of the Third STI Roadmapping Workshop, Crete, June 2009.

[10] A. Katasonov, O. Kaykova, O. Khriyenko, S. Nikitin, V. Terziyan (2008): Smart semantic middleware for the internet of things. Proceedings of the Fifth International Conference on Informatics in Control, Automation and Robotics, Funchal, Madeira, Portugal, May 2008.

[11] C. Perera, A. Zaslavsky, P. Christen, D. Georgakopoulos (2013): Context Aware Computing for The Internet of Things: A Survey. IEEE Communications Surveys \& Tutorials Journal, arXiv:1305.0982, http://arxiv.org/abs/1305.0982, 2013.

[12] International Telecommunication Union (2005): ITU Internet Reports 2005: The Internet of Things. Official Launch held at World Summit on the Information Society (WSIS) 2005 in Tunis, Tunisia, http://www.itu.int/osg/spu/publications/ internetofthings/, November 2005.

[13] H. Sundmaeker, P. Guillemin, P. Friess, S. Woelffle (2010): Vision and challenges for realising the internet of things. European Commission Information Society and Media, Technical Report, http://www.internet-of-things-research.eu/pdf/IoT_ Clusterbook_March_2010.pdf, March 2010.

[14] T. Lu, W. Neng (2010): Future Internet: The Internet of Things. 3rd International Conference on Advanced Computer Theory and Engineering (ICACTE), vol. 5, 376380, August 2010.

[15] European Commission (2008): Internet of Things in 2020: Roadmap for the future. Working Group RFID of the ETP EPOSS, May 2008.

[16] P. Guillemin, P. Friess (2009): Internet of things strategic research roadmap. The Cluster of European Research Projects, Technical Report, September 2009. 
[17] M. Minsky (1988): The Society of Mind, ISBN 0671657135, published by Simon \& Schuster, 1988.

[18] M. Minsky (2006): The Emotion Machine: Commonsense Thinking, Artificial Intelligence, and the Future of the Human Mind, ISBN 0743276647, published by Simon \& Schuster, 2006.

[19] P. Singh, T. Lin, E.T. Mueller, G. Lim, T. Perkins, W.L. Zhu (2002): Open Mind Common Sense: Knowledge Acquisition from the General Public, LNCS 2519, pp. 1223-1237, 2002.

[20] D. Lenat (1995): CYC: A Large-Scale Investment in Knowledge Infrastructure, Communications of the ACM, vol. 38, pp. 33-38, 1995.

[21] F.M. Suchanek, G. Kasneci, G. Weikum (2007): Yago: A Core of Semantic Knowledge, 16th inter-national World Wide Web conference (WWW 2007), 2007.

[22] S. Auer, J. Lehmann: What have Innsbruck and Leipzig in common? Extracting Semantics from Wiki Content. In Proceedings of ESWC'07, LNCS 4519, pp. 503-517, Springer, 2007. 